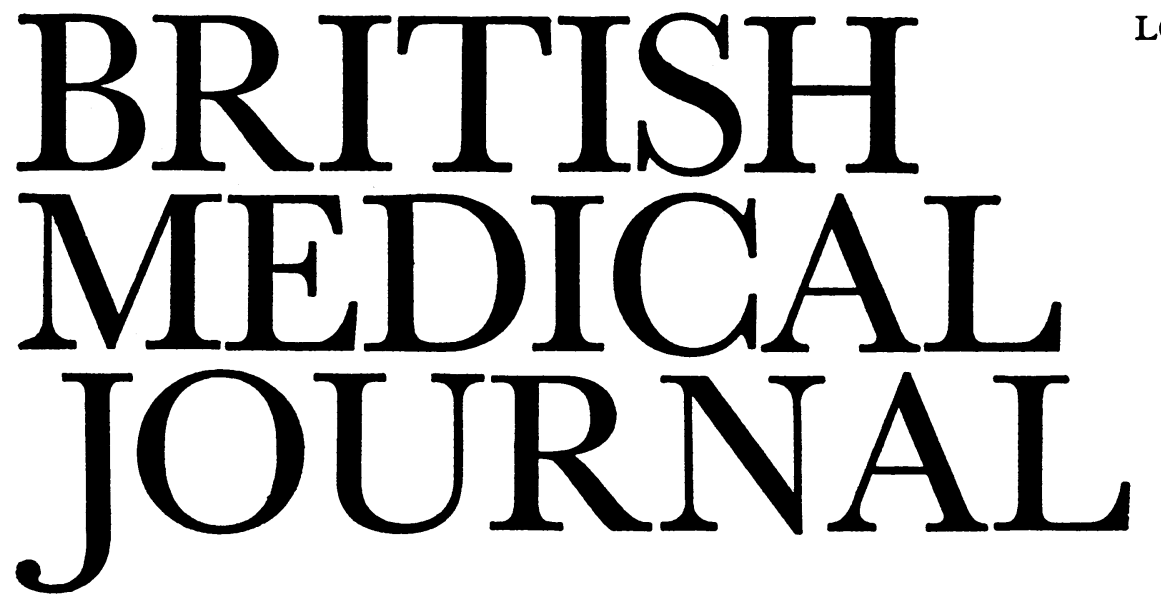

LONDON SATURDAY 19 OCTOBER 1974

\title{
Rauwolfia and Breast Cancer
}

When an accusing finger is pointed at an old drug widely used for treating a common disease a flurry of concern runs through the medical profession. Such examples in recent times include the haemolytic anaemia occasionally produced by the antihypertensive drug methyldopa ${ }^{1}$ and the excess cardiovascular mortality in diabetic patients treated with tolbutamide or phenformin. ${ }^{2}$ But when the possible toxic effect is cancer concern is even greater. Such appears to be the case with the latest offender, rauwolfia.

The principal use of rauwolfia derivatives lies in the treatment of hypertension. In Britain they are used in two types of patient. Firstly, in patients with very high blood pressure intramuscular reserpine in doses up to $5 \mathrm{mg}$ will produce an effective and predictable fall over some 12 to 18 hours. Pressure control may then be continued with some form of oral medication. Their second use is in patients with mild hypertension where, usually combined with a diuretic, rauwolfia derivatives may be given for many years. From an epidemiological study of patients in this second category the Boston Collaborative Drug Surveillance Programme has shown that the incidence of breast cancer is approximately three to four times greater in hypertensive women treated with rauwolfia preparations than in parallel control series. ${ }^{3}$ In this study the drug histories of 150 women with proved breast cancer were scrutinized. Eleven $(7.3 \%)$ of these had been taking rauwolfia compared with $13(2 \cdot 2 \%)$ of 600 surgical control patients and $13(2 \cdot 2 \%)$ of 600 medical controls. Bias in the selection of these patients seems not to be a likely cause for these differences, and other risk factors known to be associated with breast cancer were equally divided between rauwolfia patients and others. Nor could a particular rauwolfia preparation be specially indicted, though the number of patients taking each drug was small.

Based on this original observation, two other studies were conducted, one in Oxford ${ }^{4}$ and one in Finland. ${ }^{5}$ Both confirmed the Boston group's findings. In Oxford, Doll and his associates $^{4}$ examined the drug histories of 708 patients with breast cancer and 1,430 controls. Only when they excluded patients with neoplasms of pancreas, skin, body of the uterus, kidney, and nervous system from their control group (since they had prior knowledge of a possible association between rauwolfia therapy and these tumours from the original Boston data) did a significant increase in breast cancer in the women exposed to rauwolfia emerge again. In Helsinki ${ }^{5} 53$ (or $12.1 \%$ ) of 438 patients with breast cancer had been given rauwolfia preparations while in the same number of matched control groups admitted for other surgical procedures the number taking these drugs was 31 or $7 \cdot 1 \%$. In this study, as in the other two, there was evidence of association between the drug and breast cancer only in women aged more than 50 , suggesting a possible difference between pre- and postmenopausal women.

These data raise a number of important questions. Is the effect related either to drug dose or to time of exposure? The answers to both seem to be cautious affirmatives at the present time. Can one invoke the known relation between rauwolfia administration and prolactin release as a causal mechanism ? (This group of drugs will stimulate prolactin secretion from the anterior pituitary gland by depleting the stores of hypothalamic prolactin inhibitory factor, which controls the secretion of the hormone. ${ }^{6}$ Further, certain breast cancers are prolactin dependent. ${ }^{7}$ ) On this the answer seems to be a cautious negative, based on the absence of an association between breast cancer and other drugs which elevate serum prolactin such as methyldopa. Is it only breast cancer that is produced-and if so, which histological type-or are we discussing a more general carcinogenic risk to other organs too? The answer to these and many other questions remains uncertain.

But of greatest immediate concern is the course of action to be taken in women currently taking reserpine. Though the popularity of rauwolfia preparations is less in Britain than elsewhere in Europe or in the U.S.A. the problem here is still sizeable. Many physicians consider that the incidence of depression is unacceptably high in elderly patients given rauwolfia, and these results give further ammunition to reinforce the view that the drug should not be used at all in these patients. Should women taking rauwolfia have antihypertensive therapy withdrawn? The risks of untreated hypertension are widely appreciated, though there is still debate about the role of therapy in women in the very age group apparently most prone to breast cancer in these three series. Clearly each patient must be assessed separately; perhaps in those who have never suffered any of the complications of hypertension and whose pressure control is good it would be reasonable to withdraw rauwolfia and leave the patient on diuretics alone. In patients with severe hypertension, where rigorous and continued control of blood pressure is mandatory, the decision is more difficult. The possibility of breast cancer must be excluded as far as possible in all such exposed patients, and if the data so far produced are convincing to the individual doctor he has little alternative but to recommend other hypotensive therapy. This in turn poses another problem. Once rauwolfia is withdrawn, how long and what 
form should the follow up take? Firm conclusions and decisions? Probably not yet. Caution and further study? Most certainly.

\footnotetext{
1 Carstairs, K. C., et al., Lancet, 1966, 2, 133.

2 Knatterad, G. L., et al., fournal of American Medical Association, 1971 217, 777 .

3 Boston Collaborative Drug Surveillance Programme, Lancet, 1974, 2, 669

Armstrong, B., Stevens, N., and Doll, R., Lancet, 1974, 2, 672.

5 Heinonen, O. P., et al., Lancet, 1974, 2, 675.

6 Sulman, F. G., Givant, Y., and Shani-Mishkinsky, J., in Human Prolactin, Proceedings of an International Symposium, ed. J. L. Pasteels, and C. Robyn. Amsterdam, Excerpta Medica, 1973.

${ }^{7}$ Pearson, O. H., et al., Transactions of the Association of American Physicians, $1969,82,225$.
}

\section{Stroke and the Family}

The rising tide of stroke disability in Great Britain has now reached a level at which there are nearly 100,000 heavily handicapped hemiplegics. ${ }^{1}$ A recent report from the Royal College of Physicians ${ }^{2}$ has outlined the deficiencies in services available to these patients, commenting in particular on the general lack of interest in their welfare. A sense of isolation is felt as much, or more, by the relatives than by the patient in many households; only those who have experienced it fully appreciate the despair which sometimes overcomes a wife or husband who, without warning or training, has to assume for months or years a responsibility combining the skills of nurse, remedial therapist, psychologist, and speech therapist. This role, which calls for unfailing optimism, resilience, and the patience of Job, also calls for a measure of sympathetic understanding and support from community services and society which is seldom forthcoming.

The need for this kind of help for the families of stroke patients is well known to those who are specially interested in the management of hemiplegia, and it is recognized practice in stroke rehabilitation to arrange appropriate counselling to prepare relatives for the task before them when the patient leaves hospital. ${ }^{3}$ Each member of the rehabilitation team-physician, nurse, therapist, and social worker-has something special to contribute. Discussion may call for reassurance, prognosis, and a description of the plan of treatment with emphasis on the need to exploit spontaneous recovery however slow and incomplete and to use ingenuity in obtaining as much cooperation from the patient as possible. Advice may cover the details of diet, hygiene, control of bowel and bladder, manicure, the scope of self-care and activities of daily living, and adaptations in the home. It may be necessary to illustrate the daily stint of passive and self-aided movements needed to prevent contractures, the help required with balance and walking exercises, the fitting and use of appliances as part of dressing routine, and the approach to communication problems of aphasiacs. A booklet ${ }^{4}$ on the care of stroke patients is included in the Family Doctor series.

All this is essential to the restoration of social function in a hemiplegic patient, and in return relatives may divulge information they alone know which may influence the pattern of treatment and the patient's future. Details of previous history and the nature of the onset of the stroke are essential to accurate diagnosis and prognosis, much as knowledge of former personality and motivation (the "life style" of the patient) helps towards an estimate of prospects and the likely reaction to illness.
If arrangements such as these are necessary to ensure the well-being of the patients, the relatives too are entitled to special consideration of their anxieties and exigencies. They need opportunities to meet where they may find sympathetic understanding, counselling, and a sense of support through discussion, not only with professional advisers but with others who share, or have experienced, the difficulties of a family coping with hemiplegia. A forum such as this may be provided by a stroke society, but voluntary endeavour on behalf of the hemiplegic patient seems to be less enthusiastic than the support given to other kinds of handicap, and the equipment and atmosphere of a geriatric day hospital probably provides the most appropriate setting.

A programme of family stroke education ${ }^{5}$ may include occasional short talks on specific topics from professional members of the rehabilitation team, but the most successful use of their time is made when they act as a panel in periodic sessions of informal question and answer guided by a chairman or moderator able to stimulate active participation from the family members. The real test of success is the emergence of selfhelp groups willing to promote social integration of patients and relatives, to co-ordinate transport arrangements, and to initiate or encourage voluntary endeavour of one kind or another. $^{2} 3$

1 Office of Population Censuses and Surveys, Handicapped and Impaired in Great Britain, part I, by A. I. Harris, E. Cox, and C. R. W. Smith. London, H.M.S.O., 1971.

2 Royal College of Physicians of London, Report of Geriatrics Committee Working Group on Strokes. London, Royal College of Physicians, 1974.

3 Hurwitz, L. J., and Adams, G. F., British Medical fournal, 1972, 1, 94.

4 Carter, A. B., Strokes and How to Live With Them. London, British Medical Association (Family Doctor Publication), 1971.

5 Wells, R., Stroke, 1974, 5, 393.

\section{Infantile Autism}

It is now 30 years since Kanner ${ }^{1}$ presented his account of a group of 11 children with a "disorder of affective contact" characterized by an inability to develop relationships with people, delay in speech acquisition, non-communicative use of speech, repetitive manneristic behaviour with distress at any change, a good rote memory, and a normal physical appearance. Since then, there has been ample confirmation of the existence of Kanner's syndrome-infantile autism.

Rutter $^{2}$ stated recently that to make a firm diagnosis three special characteristics need to be present: firstly, a profound and general failure to develop social relationships, with a tendency to self-isolation, avoidance of direct eye-toeye gaze, and limited attachment to and differentiation of significant adults; secondly, retardation of language development, with impaired comprehension, echoing of what is heard and reversals of pronouns (calling himself "you" and not "I"); and, thirdly, ritualistic or compulsive phenomenarepetitive play with unusual objects in unusual ways.

Other characteristics not exclusive to autism include stereotyped repetitive movements of the fingers, hands, or the whole body-flicking, spinning or twirling; short attention span; hyperactive behaviour; self injuring; feeding difficulties; and delayed bowel or bladder control. Intellectual abilities range from the severely subnormal to the normal, skills often being uneven with better non-verbal skills. ${ }^{3}$

The incidence ${ }^{4}$ of infantile autism is 4.5 in 10,000. It arises in the first two years of life, and this early age of onset, together with absence of symptoms such as hallucinations or delusional 